\title{
Lymphovascular invasion represents a superior prognostic and predictive pathological factor of the duration of adjuvant chemotherapy for stage III colon cancer patients
}

\section{Jianhong Peng}

Department of Colorectal Surgery, Sun Yat-sen University Cancer Center

\section{Yuxiang Deng}

Department of Colorectal Surgery, Sun Yat-Sen University https://orcid.org/0000-0002-2873-3033

\section{Songran Liu}

Department of Pathology, Sun Yat-sen University Cancer Center

\section{Rongxin Zhang}

Department of Colorectal Surgery, Sun Yat-sen University Cancer Center

Ying Zhu

Department of Colorectal Surgery, Sun Yat-sen University Cancer Center

Zhenhai Lu

Department of Colorectal Surgery, Sun Yat-sen University Cancer Center

\section{Zhizhong Pan}

Department of Colorectal Surgery, Sun Yat-sen University Cancer Center

\section{Wenhua Fan}

Department of Colorectal Surgery, Sun Yat-sen University Cancer Center

\section{Xiaojun Wu ( $\nabla$ wuxj@sysucc.org.cn )}

Department of Colorectal Surgery, Sun Yat-sen University Cancer Center

\section{Research}

Keywords: lymphovascular invasion, perineural invasion, adjuvant chemotherapy, stage III colon cancer, prognosis

Posted Date: November 20th, 2020

DOl: https://doi.org/10.21203/rs.3.rs-108558/v1

License: (c) (i) This work is licensed under a Creative Commons Attribution 4.0 International License. Read Full License 


\section{Abstract \\ Background}

Lymphovascular invasion ( $\mathrm{LVI}$ ) and perineural invasion (PNI) can indicate poor survival outcomes in colorectal cancer, but few studies have focused on stage III colon cancer. The current study aimed to confirm the prognostic value of LVI and PNI and identify patients who could achieve benefit from a complete duration of adjuvant chemotherapy based on the two pathological factors.

\section{Methods}

We enrolled 402 consecutive patients with stage III colon cancer who receive colon tumor resection from November 2007 to June 2016 at Sun Yat-sen University Cancer Center. Survival analysis were performed by using Kaplan-Meier method with log-rank tests. Risk factors related to disease-free survival (DFS) and overall survival (OS) were identified through Cox proportional hazards analysis.

\section{Results}

A total of 141 (35.1\%) patients presented with LVI, and 108 (26.9\%) patients with PNI. The LVI-positive group was associated with poorer 3-year DFS ( $86.5 \%$ vs. $76.3 \%, \mathrm{P}=0.001)$ and OS $(96.0 \%$ vs. $89.1 \%, \mathrm{P}=$ 0.003 ) rates compared with the LVI-negative group. The PNI-positive group showed a worse survival outcome compared with the PNI-negative group in 3-year DFS rate $(72.5 \%$ vs. $86.7 \%, \mathrm{P}<0.001)$. Moreover, LVI-positive group present better 3-year DFS and OS rate in patients completing 6-8 cycles of adjuvant chemotherapy than those less than 6 cycles (3-year DFS: $80.0 \%$ vs. $64.9 \%, P=0.019$; 3-year OS: $93.2 \%$ vs. $76.3 \%, P=0.002)$.

\section{Conclusions}

LVI is a superior prognostic factor to PNI in stage III colon cancer patients undergoing curative treatment. Furthermore, LVI also represents an effective indicator for adjuvant chemotherapy duration.

\section{Background}

To date, the combination of curative surgery and oxaliplatin-based chemotherapy are well recommended as the classical treatment strategy for stage III colon cancer [1, 2]. A 6-month duration of adjuvant chemotherapy was previously recommended for all stage III colon cancer patients [3, 4]. However, two major problems remain to be solved in clinical practice. In fact, $20-40 \%$ of patients fail to benefit from adjuvant chemotherapy and ultimately develop postoperative metastases [5, 6]. Moreover, chemotherapyrelated toxicity, especially oxaliplatin-based sensory neurotoxicity, caused $50 \%$ of patients to be unable to finish the full planned duration of adjuvant chemotherapy [7]. Therefore, the treatment duration of 
adjuvant chemotherapy warrants individual management instead of a fixed course model. Recently, International Duration Evaluation of Adjuvant Therapy (IDEA) trial introduced risk definitions for stage III colon cancer to guide the duration of adjuvant chemotherapy. The final results indicated that 3-month XELOX adjuvant chemotherapy appeared to be sufficient for low-risk patients (T1-3 N1 disease) but not high-risk patients (T4, N2, or both disease) [8]. Although the TNM stage is important for colon cancer management, it seems not to be sufficient to determine the specific risk patiens with stage III colon cancer will actually benefit from adjuvant chemotherapy. Herein, other pathological factors are needed to aid in risk stratification and to select patients who need adjuvant treatment.

Current guidelines note that two tumor specific parameters including lymphovascular invasion (LVI) and perineural invasion (PNI) should be reported in the pathological stage for colon cancer [3, 9]. LVI is recognized that tumor cells involved in small endothelium-lined lymphatic or vascular channels, which indicated as an early and obligatory step of tumor metastasis $[10,11]$. PNI is defined as a pathologic process of tumor growth within the nerves and nerve sheaths and is a pathological marker for a more aggressive tumor phenotype $[12,13]$. Accumulating evidence has well demonstrated that the presence of either or both pathological factors indicates a poor survival outcome in colorectal cancer (CRC) $[14,15]$. Currently, most studies have focused on the investigating the prognostic role of the two pathological factors in stage I and stage II CRC and identified both as indications for adjuvant chemotherapy $[16,17]$. However, only a limited number of studies reported the prognostic value of LVI and PNI in stage III colon cancer $[18,19]$. In fact, the actual prognostic effect of LVI and PNI remains unclear in stage III colon cancer patients undergoing curative treatment. In addition, evidence on whether LVI and PNI could serve as markers for the duration of adjuvant chemotherapy is still lacking.

To address these two issues, the current study aimed to demonstrate the prognostic effect of LVI and PNI in stage III colon cancer patients receiving curative surgery followed by adjuvant chemotherapy. Subsequently, we aimed to identify the specific group of patients who could actually benefit from a full duration of adjuvant chemotherapy according to presence of LVI and PNI.

\section{Materials And Methods}

\section{Patients}

A total of 402 consecutive patients with stage III colon cancer who underwent primary tumor resection between November 2007 and June 2016 at Sun Yat-sen University Cancer Center were included in this retrospective study. The patients were enrolled according to the following criteria: (1) pathologically diagnosed as colon adenocarcinoma; (2) colon tumor curative resection; (3) adjuvant chemotherapy with the XELOX regimen (oxaliplatin plus capecitabine); (4) complete pathological data with definite LVI and PNI statuses; (5) no preoperative anticancer treatment; (6) American Society of Anesthesiologists class III; and (7) postoperative follow-up at least 3 months after delivery of the first cycle of chemotherapy. The clinical information including demographics, tumor characteristics, treatment details and follow-up data were carefully collected from the electronic medical record system. Right-sided colon cancer was defined 
as the tumor locating in cecum, ascending, hepatic flexure, and transverse colon, whereas left-sided colon cancer was recognized as the tumor in splenic flexure, descending, and sigmoid colon. The current study was conducted based on the ethical standards of the World Medical Association Declaration of Helsinki. Ethics approval from Ethics Committees of Sun Yat-sen University Cancer Center was waived because of the nature of retrospective study, and the patient data were kept confidentially. All patient data were documented confidentially.

\section{Treatments}

All the patients underwent curative resection of the colon tumor by performing standard complete mesocolic excision and regional lymphadenectomy. The initial adjuvant chemotherapy were performed 3-8 weeks for all the patients after colon tumor resection. The XELOX regimen was administered as a 3week cycle chemotherapy as $130 \mathrm{mg} / \mathrm{m}^{2}$ oxaliplatin on day 1 combined with $1,000 \mathrm{mg} / \mathrm{m}^{2}$ capecitabine twice daily on days 1-14 at an interval of 7 days. The continue administration of the XELOX regimen of adjuvant chemotherapy depended on patient's general status, the toxicity of the chemotherapy or the patient's tolerance to the subsequent cycle of chemotherapy.

\section{Pathologic analysis}

Each tumor resection specimen was reviewed by two independent pathologists (Songran Liu and Shixun $\mathrm{Lu}$ ). All cases were pathologically staged referring to the 8th edition of the American Joint Committee on Cancer (AJCC) staging system. Hematoxylin and eosin staining was used to evaluate the LVI status without other special stains. LVI was diagnosed as the presence of tumor cells within the small endothelium-lined lymphatic or vascular channels [10]. PNI was diagnosed as tumor invasion in, around, and through nerves and nerve sheaths (Fig. 1) [12]. In addition, the statuses of proximal and distal margins, lymph node metastasis and tumor differentiation were assessed in line with current guidelines $[3,9]$.

\section{Postoperative follow-up}

The follow-up were conducted every 3 months for the first 2 years and then semiannually for subsequent 3 years after surgery through clinical visits. The clinical visit items included abdomen examinations, detection of serum carcinoembryonic antigen (CEA) and carbohydrate antigen 19 - 9 (CA19-9), chest/abdominal/pelvic CT and colonoscopy. Disease-free survival (DFS) was the interval from the date of tumor resection to the date of disease recurrence, death or the last follow-up. Overall survival (OS) was the interval from the date of tumor resection to the date of death from any cause or the last follow-up. The final follow-up visit conducted in July 2019.

\section{Statistical analysis}

Statistical analyses were conducted through SPSS 20.0 software (IBM, Chicago, IL, USA) and GraphPad Prism 7 software (GraphPad Software, Inc., San Diego, CA, USA). Continuous variables are presented as the median (range), while categorical variables are presented as percentages, which were compared by using the chi-square $(\chi 2)$ test. The Kaplan-Meier curve was applied to calculate the survival rates, and 
the differences in survival of serval group patients were subsequently compared by using the log-rank test. A multivariate Cox proportional hazards was developed to identify the independent risk factors by including the parameters whose $P$ value was less than 0.05 in the univariate analysis. The Hazard ratios (HRs) and 95\% confidence intervals (Cls) were finally generated. The statistical tests performed above were two sided, and a $P$ value less than 0.05 was considered significant.

\section{Results}

\section{Patient characteristics}

The detail clinicopathologic information of the total patients were shown in Table 1. The median age of all patients was 56 years (range, $19-78$ years), and $60.7 \%$ of the patients were male. The median tumor size was $4.0 \mathrm{~cm}$ (range, $1.0-13.0 \mathrm{~cm}$ ). The median number of retrieved lymph nodes was 16 (range 268), and the median number of metastatic lymph nodes was 2 (range 1-23). Of all the patients, 87 (21.6\%) had positive LVI alone, 54 (13.4\%) had positive PNI alone, 54 (13.4\%) had both positive LVI and $\mathrm{PNI}$, and 207 (51.5\%) had both negative LVI and PNI. Accordingly, 141 (35.1\%) patients belonged to the LVI-positive group, and 108 (26.9\%) patients belonged to the PNI-positive group. Regarding adjuvant chemotherapy, the median cycle of the XELOX regimen was 6 (range, 1-8). 
Table 1

Clinical and pathological information of total patients in current study

\section{Characteristic}

Age (years)

Tumor site

Sex

Tumor site

Tumor size (cm)

T stage

N stage

TNM stage

Tumor differentiation

LVI

$$
\leq 60
$$$$
>60
$$

Female

Male

Cecum

Ascending colon

Hepatic flexure

Transverse colon

Splenic flexure

Descending colon

Sigmoid colon

$$
\leq 4.0
$$$$
>4.0
$$

1

2

3

4

1

2

IIIA

IIIB

IIIC

Well/moderate

Poor

Positive
№. (\%)

$263(65.4)$

139 (34.6)

158 (39.3)

$244(60.7)$

$19(4.7)$

71 (17.7)

$38(9.5)$

42 (10.4)

7 (1.7)

32 (8.0)

193 (48.0)

211 (52.5)

191 (47.5)

2 (0.5)

15 (3.7)

229 (57.0)

156 (38.8)

295 (73.4)

107 (26.6)

$11(2.7)$

$189(47.0)$

$202(50.2)$

$255(63.4)$

$147(36.6)$

$141(35.1)$

Abbreviations: CEA, carcinoembryonic antigen; CA19-9, cancer antigen 19 - 9; LVI, lymphovascular invasion; PNI, perineural invasion; TNM stage, clinical tumor-node-metastasis stage. 


\begin{tabular}{|lll|}
\hline Characteristic & & No. (\%) \\
\hline PNI & Negative & $261(64.9)$ \\
\hline & Positive & $108(26.9)$ \\
\hline Preoperative CEA (ng/ml) & Negative & $294(73.1)$ \\
\hline & $\leq 5$ & $225(56.0)$ \\
\hline Preoperative CA19-9 (U/ml) & $>5$ & $177(44.0)$ \\
\hline & $\leq 35$ & $323(80.3)$ \\
\hline Adjuvant chemotherapy cycle & $>35$ & $79(19.7)$ \\
\hline & $<6$ & $96(23.9)$ \\
\hline $\begin{array}{l}\text { Abbreviations: CEA, carcinoembryonic antigen; CA19-9, cancer antigen 19 - 9; LVI, lymphovascular } \\
\text { invasion; PNI, perineural invasion; TNM stage, clinical tumor-node-metastasis stage. }\end{array}$ \\
\hline
\end{tabular}

\section{The relationship of LVI and PNI with clinicopathological features}

As shown in Table 2, the presence of both $L V I$ and $P N I$ was positively related to $N 2$ stage $(P=0.025 ; P=$ $0.036)$ and poorer tumor differentiation $(P=0.002 ; P=0.014)$. Moreover, $L V I$ was associated with $T 4$ stage $(P<0.001)$. The large tumor size was more frequently found in the patients with PNI negative tumor $(P=0.006)$. The differences of other parameters between the groups did not show statistical significance. 
Table 2

Relationships between LVI as well as PNI and patient characteristics.

Characteristic

LVI

Positive, $\mathrm{n} \quad$ Negative, $\mathrm{n}$

$=141(\%) \quad=261(\%)$
$\mathbf{P}$

value
PNI

Positive, Negative, $P$ $\mathrm{n}=108 \quad \mathrm{n}=\mathbf{2 9 4}(\%) \quad$ value

(\%)

Age (years)

$\leq 60$

$98(69.5)$

$165(63.2)$

0.206

96 (36.8)

$43(30.5)$

Sex

Female

57 (40.4)

101 (38.7)

0.735

$160(61.3)$

$84(59.6)$

Tumor site

Right-sided colon
Left-sided colon
Tumor size (cm)

$\leq 4.0$

$82(58.2)$

129 (49.4)

0.094

$132(50.6)$

0.178

47 (43.5)

$123(41.8)$

0.762

$>4.0$

$59(41.8)$

$157(60.2)$

61 (56.5) $171(58.2)$

T stage

$1-3$

$67(47.5)$

179 (68.6)

$<$

0.001

$82(31.4)$

$74(52.5)$

$82(31.4)$

$47(43.5) \quad 109(37.1)$

$\mathrm{N}$ stage

2

$94(66.7)$

$201(77.0)$

0.025

$71(65.7)$

224 (76.2)

0.036

2

47 (33.3)

$60(23.0)$

$37(34.3) \quad 70(23.8)$

\section{Tumor}

differentiation

Well/moderate

75 (53.2)

$180(69.0)$

0.002

58 (53.7)

$197(67.0)$

0.014

Poor

$66(46.8)$

$81(31.0)$

$50(46.3)$

97 (33.0)

\section{Preoperative CEA}

( $\mathrm{ng} / \mathrm{ml})$

Abbreviations: CEA, carcinoembryonic antigen; CA19-9, cancer antigen 19 - 9; LVI, lymphovascular invasion; PNI, perineural invasion. 


\begin{tabular}{|lllllll}
\hline Characteristic & LVI & \multicolumn{5}{l}{ PNI } \\
\hline$\leq 5$ & $75(53.2)$ & $150(57.5)$ & 0.409 & $59(54.6)$ & $166(56.5)$ & 0.743 \\
\hline$>5$ & $66(46.8)$ & $111(42.5)$ & & $49(45.4)$ & $128(43.5)$ & \\
\hline $\begin{array}{l}\text { Preoperative } \\
\text { CA19-9 (U/ml) }\end{array}$ & & & & & & \\
\hline$\leq 35$ & $113(80.1)$ & $210(80.5)$ & 0.939 & $87(80.6)$ & $236(80.3)$ & 0.949 \\
$>35$ & $28(19.9)$ & $51(19.5)$ & & $21(19.4)$ & $58(19.7)$ & \\
\hline $\begin{array}{l}\text { Adjuvant } \\
\text { chemotherapy } \\
\text { cycles }\end{array}$ & & & & & & \\
\hline$<6$ & $35(24.8)$ & $61(23.4)$ & 0.745 & $21(19.4)$ & $75(25.5)$ & 0.206 \\
\hline $6-8$ & $106(75.2)$ & $200(76.6)$ & & $87(80.6)$ & $219(74.5)$ & \\
\hline
\end{tabular}

Abbreviations: CEA, carcinoembryonic antigen; CA19-9, cancer antigen 19 - 9; LVI, lymphovascular invasion; PNI, perineural invasion.

\section{Prognostic value of LVI and PNI for DFS and OS}

After a median postoperative follow-up duration of 56 months (range, 7-114 months), 77 (19.1\%) patients developed disease recurrence, and $39(9.7 \%)$ patients ultimately occurred cancer-related mortality. Among these patients, 39.0\% (30/77) had liver metastases, 36.4\% (28/77) had lung metastases, $39.0 \%$ (30/77) had abdominal pelvic metastases, and $5.2 \%(4 / 77)$ had metastases to other organs. The 3-year DFS and OS rates were $82.9 \%$ and $93.6 \%$ in total enrolled patient in this study.

Kaplan-Meier analysis indicated that LVI-positive group showed the significantly worse 3-year DFS and OS rates compared with those in the LVI-negative group (DFS: $76.3 \%$ vs. $86.5 \%, P=0.001$, Fig. $2 \mathrm{~A}$; OS: $89.1 \%$ vs. $96.0 \%, P=0.016$, Fig. $2 B$ ). PNI-positive group presented significantly lower 3-year DFS rate by comparison of those with the PNI-negative group ( $72.5 \%$ vs. $86.7 \%, \mathrm{P}<0.001$, Fig. $2 \mathrm{C}$ ), while the 3-year OS rate was comparable between the two groups $(90.5 \%$ vs. $94.7 \%, P=0.134$, Fig. $2 \mathrm{D})$. Patients with the concurrent presence of LVI and PNI had the worst 3-year DFS (65.5\% vs. $81.3 \%$ vs. $88.4 \%, \mathrm{P}<0.001$; Fig. $2 E)$ and $O S(84.6 \%$ vs. $93.5 \%$ vs. $96.0 \%, P=0.010$; Fig. $2 F)$ rates among those with either the presence of $\mathrm{LVI}$ and $\mathrm{PNI}$ and the absence of $\mathrm{LVI}$ and PNI.

\section{Identification of the independent prognostic factors}

The results of univariate and multivariate analyses are summarized in Table 3. The univariate analysis result revealed that male sex, N2 stage, poorly differentiated tumor histology, LVI, PNI, preoperative CEA > $5 \mathrm{ng} / \mathrm{ml}$ and preoperative CA19-9>35 U/ml were associated with unfavorable DFS, while right-sided colon cancer, T4 stage, N2 stage, LVI, preoperative CA19-9 > $35 \mathrm{U} / \mathrm{ml}$ and adjuvant chemotherapy less than 6 cycles were associated with unfavorable OS. In addition, multivariate result analysis showed that male sex (HR, 1.906; 95\% Cl, 1.180-3.077; P = 0.008), LVI (HR, 1.828; 95\% Cl, 1.182-2.825; P = 0.007), PNI 
$(\mathrm{HR}, 1.921 ; 95 \% \mathrm{Cl}, 1.238-2.981 ; \mathrm{P}=0.004)$, and preoperative CEA > $5 \mathrm{ng} / \mathrm{ml}(\mathrm{HR}, 1.756 ; 95 \% \mathrm{Cl}, 1.142-$ 2.701; $P=0.010$ ) were the independent predictive factors for unfavorable $D F S$, while right-sided colon cancer $(\mathrm{HR}, 0.427 ; 95 \% \mathrm{Cl}, 0.223-0.815 ; \mathrm{P}=0.010)$, T4 stage $(\mathrm{HR}, 2.777 ; 95 \% \mathrm{Cl}, 1.435-5.374 ; \mathrm{P}=0.002)$ and preoperative CA19-9 > $35 \mathrm{U} / \mathrm{ml}(\mathrm{HR}, 2.472 ; 95 \% \mathrm{Cl}, 1.279-4.780 ; \mathrm{P}=0.007)$ were the independent predictive factors for unfavorable OS. 
Table 3

Univariate and multivariate analyses for identifying prognostic factors.

DFS OS

\begin{tabular}{llll}
$\begin{array}{l}\text { Univariate } \\
\text { analysis }\end{array}$ & $\begin{array}{l}\text { Multivariate } \\
\text { analysis }\end{array}$ & $\begin{array}{l}\text { Univariate } \\
\text { analysis }\end{array}$ & $\begin{array}{l}\text { Multivariate } \\
\text { analysis }\end{array}$ \\
\hline
\end{tabular}

$\begin{array}{llllllll}\text { HR }(95 \% & \mathbf{P} & \text { HR }(95 \% & \mathbf{P} & \text { HR }(95 \% & \mathbf{P} & \text { HR } & \mathbf{P}\end{array}$

$\begin{array}{lllll}\mathrm{Cl}) & \text { value } \mathrm{Cl}) & \text { value } & \mathrm{Cl}) & \text { value } \\ (95 \% & \text { value }\end{array}$

$\mathrm{Cl})$

Age (years)

$>60$ vs. $\leq 60$

$\begin{array}{ll}1.435 & 0.102 \\ (0.931- & \\ 2.211) & \end{array}$

1.592

0.150

$(0.845-$

2.998)

Sex

Male vs. Female

$\begin{array}{lllll}1.808 & 0.014 & 1.906 & 0.008 & 1.567 \\ (1.127- & & (1.180- & & (0.794- \\ 2.900) & & 3.077) & & 3.095)\end{array}$

0.196

2.900)

$0.944 \quad 0.792$

(0.614-

0.415

(0.218-

0.792 )

0.008

0.427

$(0.223-$

0.010

vs. Right-sided

1.451)

Tumor size (cm)
$>4.0$ vs. $\leq 4.0$
0.797
0.301
0.783
0.453
(0.518-
(0.414-
1.483)

\section{T stage}

4 vs. $1-3$

$\begin{array}{ll}1.218 & 0.367 \\ (0.793- & \\ 1.872) & \end{array}$

2.578
$(1.334-$
$4.985)$

0.005

2.777

(1.435-

5.374)

0.002

4.985)

$\mathrm{N}$ stage

2 vs. 1

$\begin{array}{ll}1.637 & 0.029 \\ (1.052- & \\ 2.547) & \end{array}$

0.085

2.316

0.009

0.060

2.547)

(1.234-

4.349)

Tumor differentiation

\begin{tabular}{|c|c|c|c|c|}
\hline $\begin{array}{l}\text { Poor vs. } \\
\text { Well/moderate }\end{array}$ & $\begin{array}{l}1.576 \\
(1.025- \\
2.424)\end{array}$ & 0.038 & 0.241 & $\begin{array}{l}1.330 \\
(0.695- \\
2.546)\end{array}$ \\
\hline
\end{tabular}

Abbreviations: $\mathrm{HR}$, hazard ratio; $\mathrm{Cl}$, confidence interval; $\mathrm{LVI}$, lymphovascular invasion; $\mathrm{PNI}$, perineural invasion; CEA, carcinoembryonic antigen; CA19-9, cancer antigen 19-9. 


\section{DFS}

OS

\section{LVI}

\begin{tabular}{|c|c|c|c|c|c|c|}
\hline $\begin{array}{l}\text { Positive vs. } \\
\text { Negative }\end{array}$ & $\begin{array}{l}1.985 \\
(1.297- \\
3.037)\end{array}$ & 0.002 & $\begin{array}{l}1.828 \\
(1.182- \\
2.825)\end{array}$ & 0.007 & $\begin{array}{l}2.122 \\
(1.132- \\
3.979)\end{array}$ & 0.019 \\
\hline
\end{tabular}

\section{PNI}

$\begin{array}{lllllll}\text { Positive vs. } & 2.250 & < & 1.921 & 0.004 & 1.641 & 0.138 \\ \text { Negative } & (1.463- & 0.001 & (1.238- & & (0.853- & \\ & 3.461) & & 2.981) & & 3.159) & \end{array}$

\section{Preoperative CEA}

(ng/ml)

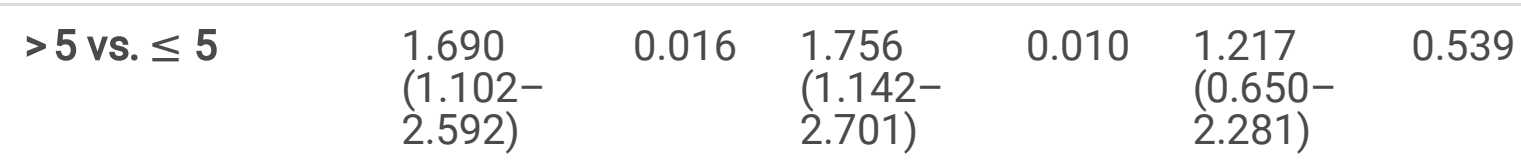

\section{Preoperative \\ CA19-9 (U/ml)}

\begin{tabular}{|c|c|c|c|c|c|c|}
\hline$>35$ vs. $\leq 35$ & $\begin{array}{l}1.690 \\
(1.047- \\
2.727)\end{array}$ & 0.032 & 0.115 & $\begin{array}{l}2.447 \\
(1.271- \\
4.711)\end{array}$ & 0.007 & $\begin{array}{l}2.472 \\
(1.279- \\
4.780)\end{array}$ \\
\hline
\end{tabular}

\section{Adjuvant}

chemotherapy

cycles
$6-8$ vs. $<6$
0.693
0.129
0.461
(0.239-
0.021
0.054
(0.432-
0.888 )

Abbreviations: $\mathrm{HR}$, hazard ratio; $\mathrm{Cl}$, confidence interval; $\mathrm{LVI}$, lymphovascular invasion; $\mathrm{PNI}$, perineural invasion; CEA, carcinoembryonic antigen; CA19-9, cancer antigen $19-9$.

Prognostic analysis the different adjuvant chemotherapy duration with respect to the statuses of LVI and PNI

Among the LVI-positive group, the patients completing 6-8 cycles of adjuvant chemotherapy presented significantly better 3-year DFS and OS rates than those who completed less than 6 cycles (DFS: $80.0 \%$ vs. $64.9 \%, P=0.019$, Fig. 3A; OS: $93.2 \%$ vs. $76.3 \%, P=0.002$, Fig. 3B). For the LVI-negative group, the 3-year DFS or OS rate was comparable between patients who completed 6-8 cycles of adjuvant chemotherapy and those who completed less than 6 cycles (DFS: $86.7 \%$ vs. $85.2, P=0.915 ;$ OS: $96.4 \%$ vs. $94.7 \%, P=$ $0.921)$.

However, despite the PNI status, no significantly difference was observed in the 3-year DFS or OS rate whatever the patients completed 6-8 cycles of adjuvant chemotherapy or less than 6 cycles (Fig. 4). 


\section{Discussion}

It is well known that tumor invasion and the number of metastatic lymph nodes are two vital pathological parameters that can be applied to identify the recurrence risk in stage III colon cancer [20]. In addition to TNM staging, the companion stage diagnosis characterized by other specific pathologic parameters warrant to refine stage III colon cancer risk classifications. In the current study, we performed an assessment of LVI and PNI in tumor specimens and further demonstrated their prognostic value for stage III colon cancer. As a result, two important findings from this study were noted: (1) LVI and PNI are two important pathological factors for predicting 3-year DFS in patients with stage III colorectal cancer; and (2) LVI is a better therapeutic indicator than PNI, in that LVI can also serve as a effective predictive factor for efficacy of sufficient duration of adjuvant chemotherapy.

Our data showed that the incidences of LVI and PNI among the 402 patients were $35.1 \%$ and $26.9 \%$, respectively, which were higher than those described in previous reports [21, 22]. In fact, the frequency of LVI and PNI is stage dependent and indicates the likelihood of lymph node involvement. Previous studies have noted that PNI involvement was present in only $9.5 \%$ of stage I and II CRC tumors compared with $26.3 \%$ of stage III tumors and $36.6 \%$ of stage IV CRC tumors. Similarly, the presence of LVI also increased from $5.5 \%$ in stage I tumor to $24.4 \%$ in stage IV tumor $[23,24]$. In addition, another study revealed that the presence of LVI or PNI was the independent risk factor for lymph node metastasis in colorectal cancer [25]. Similarly, our findings revealed that the presence of LVI and PNI had a positive association with advanced $\mathrm{N}$ stage, which indicates an adverse clinical course in stage III colon cancer. Thus, these findings suggest that the LVI and PNI was an indicator for more extensive surgical tumor resection.

To our knowledge, the unfavorable prognostic impact of LVI and PNI in lymph node-negative colorectal cancer patients has been well identified $[16,17,22]$. Therefore, current practice guidelines suggest that adjuvant chemotherapy was recommended for stage II colon cancer patients with poor prognostic characteristics, including PNI and LVI. Recently, Zhong JW et al revealed that LVI was an indicator of more aggressive biological behavior and a unfavorable prognosis in patients with stage III colorectal cancer [18]. However, there were two limitations to the study by Zhong JW et al that could not confirm the prognostic value of LVI for stage III colon cancer. The study included not only colon cancer patients but also rectal cancer patients. Previous studies have revealed different recurrence patterns between LVIpositive colon cancer and LVI-positive rectal cancer, which indicating that the prognostic properties of LVI existed discrepancies in colon cancer and rectal cancer [26]. Moreover, information on postoperative treatment is unavailable, which underestimates the prognostic value of LVI. Unlike the study by Zhong JW et al, our present study focused only on stage III colon cancer and performed unified curative resection followed by adjuvant chemotherapy with the XELOX regimen. Subsequently, we further confirmed the presence of PNI, which indicated poor 3-year DFS and OS in these patients. Fujita S et al were the first to report that PNI-positive group presented the significantly worse DFS rate of compared with that of the PNI-negative group in stage III colon cancer, despite of adjuvant chemotherapy administration [19]. Our study revealed that the presence of PNI not only indicated worse 3-year DFS compared to the absence of PNI but was also an independent risk factor. Moreover, we also identified a subgroup of patients with the 
presence of both LVI and PNI showed the worst 3-year DFS and OS rates, revealing the prognostic superposition of the two pathological parameters in colon cancer.

Here, LVI and PNI, serving as unfavorable prognostic factors in colon cancer, might mainly

attribute to the aggressive tumor type. Our findings indicate that LVI and PNI are significantly related to a poor pathological differentiation and an advanced tumor stage. Similarly, previous data have shown that both LVI and PNI were closely related to the aggressive tumor features characterized by a poor pathological differentiation and an advanced tumor stage $[27,28]$. Jiang $\mathrm{HH}$ et al revealed that the presence of LVI were correlated with genomic alterations activating aggressive tumor behavior, such as angiogenesis, epithelial-mesenchymal transition, and matrix remodeling [24]. Kim JC et al reported that the existence of PNI was closely associated with the expression of gelsolin, which promoted tumor cell proliferation and migration by degrading extracellular matrix and subsequently contributes to the systemic recurrence of colorectal cancer [29]. We believe that understanding the potential mechanisms underlying this association might help improve future therapeutic strategies to inhibit the metastatic spread of cancer with LVI and PNI.

Interestingly, LVI exhibited both prognostic value and predictive power in response to adjuvant chemotherapy, while PNI had only prognostic value in 3-year DFS for stage III colon cancer. A recent study found that PNI is able to serve as a prognostic indicator but not a predictive indicator for adjuvant chemotherapy efficacy for colon cancer [30]. Accordingly, the presence of LVI is a more important pathological risk factor than PNI. Our findings revealed that treatment benefits from the full planned duration of adjuvant chemotherapy were only observed in patients in LVI-positive group but not in the LVInegative group. Therefore, a novel strategy based on the presence of LVI can be developed for individual management of adjuvant chemotherapy for stage III colon cancer. Once patients are found to have LVI, a sufficient duration of adjuvant chemotherapy should be generally recommended, whereas for patients without LVI, an full course of adjuvant chemotherapy is better to be avoided to minimize chemotherapyrelated toxicity.

Several limitations to the current study were acknowledged. First, this retrospective study was performed with an uncontrolled methodology by including a limited number of patients at a single cohort. Although our study initially indicated the potential prognostic value of $\mathrm{LVI}$ and $\mathrm{PNI}$, the findings are necessary to be validated in a prospective, multicenter clinical trial with a large population in the future. Second, the median 56 months follow-up duration had insufficient power to calculate 5-year survival outcomes, which might result in a misestimation of the effect of LVI and PNI on OS. Additionally, tumor molecular markers, such as the microsatellite status, the CpG island methylator phenotype (CIMP) status, driver gene mutations, such as KRAS and BRAF, and tumor immune microenvironment, have been linked to different recurrence risks of stage III colon cancer [31,32]. The above molecular data were unavailable in current study. Thus, it is necessary to include molecular prognostic markers for risk stratification in further studies. 


\section{Conclusion}

Our study confirms that LVI represents a superior prognostic factor to PNI for stage III colon patients undergoing curative resection followed by adjuvant chemotherapy. Moreover, our findings also indicate the predictive value of LVI as an efficacy indicator for adjuvant chemotherapy duration.

\section{Declarations}

\section{Ethics approval and consent to participate}

Ethics approval from Ethics Committees of Sun Yat-sen University Cancer Center was waived because of the nature of retrospective study, and the patient data were kept confidentially. All patient data were documented confidentially.

\section{Consent for publication}

Not applicable

\section{Availability of data and materials}

The authenticity of this article has been validated by uploading the key raw data onto the Research Data Deposit public platform (http://www.researchdata.org.cn), with the approval number as RDDA2019001198.

\section{Competing interests}

The authors declare that they have no competing interests

\section{Funding}

The study was funded by the grants from Fundamental Research Funds for the Central Universities (No. 19ykyjs77), National Natural Science Foundation of China (No. 81502459, No. 81772595 and No. 81871991), the Guangzhou Science and Technology Plan Projects (Health Medical Collaborative Innovation Program of Guangzhou)(grant No. 201803040019 and No. 201400000001-4), Science and Technology Planning Project of Guangdong Province (No.2013B090800047) and Science and Technology Planning Project of Guangzhou city (No. 201704030101).

\section{Authors' contributions}

JP, YD, SL made substantial contributions to the design of the study. JP, YD drafted the manuscript. JP, YD, SL, RZ, YZ, ZL, ZP, WF, XW planned, coordinated, and conducted the study. YD contributed to data management. All authors contributed to the implementation of the study, were involved in revising the manuscript critically. All authors read and approved the final manuscript. 
We deeply appreciate Dr. Shixun Lu of the Department of Pathology in Sun Yat-sen University Cancer Center, who assisted with the pathological assessment of LVI and PNI.

\section{References}

1. Schmoll HJ, Twelves C, Sun W, O'Connell MJ, Cartwright T, McKenna E, et al. Effect of adjuvant capecitabine or fluorouracil, with or without oxaliplatin, on survival outcomes in stage III colon cancer and the effect of oxaliplatin on post-relapse survival: a pooled analysis of individual patient data from four randomised controlled trials. LANCET ONCOL. [Journal Article; Multicenter Study; Randomized Controlled Trial; Research Support, N.I.H., Extramural; Research Support, Non-U.S. Gov't]. 2014 2014-12-01;15(13):1481-92.

2. Andre T, Boni C, Navarro M, Tabernero J, Hickish T, Topham C, et al. Improved overall survival with oxaliplatin, fluorouracil, and leucovorin as adjuvant treatment in stage II or III colon cancer in the MOSAIC trial. J CLIN ONCOL. [Clinical Trial, Phase III; Journal Article; Multicenter Study; Randomized Controlled Trial; Research Support, Non-U.S. Gov't]. 2009 2009-07-01;27(19):3109-16.

3. Labianca R, Nordlinger B, Beretta GD, Mosconi S, Mandala M, Cervantes A, et al. Early colon cancer: ESMO Clinical Practice Guidelines for diagnosis, treatment and follow-up. ANN ONCOL. [Journal Article; Practice Guideline]. 2013 2013-10-01;24 Suppl 6:i64-72.

4. Benson AR, Venook AP, Bekaii-Saab T, Chan E, Chen YJ, Cooper HS, et al. Colon cancer, version 3.2014. J Natl Compr Canc Netw. [Guideline; Journal Article]. 2014 2014-07-01;12(7):1028-59.

5. Taieb J, Tabernero J, Mini E, Subtil F, Folprecht G, Van Laethem JL, et al. Oxaliplatin, fluorouracil, and leucovorin with or without cetuximab in patients with resected stage III colon cancer (PETACC-8): an open-label, randomised phase 3 trial. LANCET ONCOL. [Clinical Trial, Phase III; Journal Article; Multicenter Study; Randomized Controlled Trial; Research Support, Non-U.S. Gov't]. 2014 2014-0701;15(8):862-73.

6. Saltz LB, Niedzwiecki D, Hollis D, Goldberg RM, Hantel A, Thomas JP, et al. Irinotecan fluorouracil plus leucovorin is not superior to fluorouracil plus leucovorin alone as adjuvant treatment for stage III colon cancer: results of CALGB 89803. J CLIN ONCOL. [Journal Article; Randomized Controlled Trial; Research Support, N.I.H., Extramural]. 2007 2007-08-10;25(23):3456-61.

7. Lonardi S, Sobrero A, Rosati G, Di Bartolomeo M, Ronzoni M, Aprile G, et al. Phase III trial comparing 3-6 months of adjuvant FOLFOX4/XELOX in stage II-III colon cancer: safety and compliance in the TOSCA trial. ANN ONCOL. [Journal Article; Published Erratum]. 2017 2017-12-01;28(12):3110.

8. Grothey A, Sobrero AF, Shields AF, Yoshino T, Paul J, Taieb J, et al. Duration of Adjuvant Chemotherapy for Stage III Colon Cancer. N Engl J Med. [Comparative Study; Equivalence Trial; Journal Article; Research Support, N.I.H., Extramural; Research Support, Non-U.S. Gov't]. 2018 201803-29;378(13):1177-88.

9. Benson AB, Venook AP, Al-Hawary MM, Cederquist L, Chen YJ, Ciombor KK, et al. NCCN Guidelines Insights: Colon Cancer, Version 2.2018. J Natl Compr Canc Netw. [Journal Article]. 2018 2018-0401;16(4):359-69. 
10. Harris El, Lewin DN, Wang HL, Lauwers GY, Srivastava A, Shyr Y, et al. Lymphovascular invasion in colorectal cancer: an interobserver variability study. AM J SURG PATHOL. [Journal Article; Research Support, N.I.H., Extramural; Research Support, Non-U.S. Gov't]. 2008 2008-12-01;32(12):1816-21.

11. Achen MG, Stacker SA. Tumor lymphangiogenesis and metastatic spread-new players begin to emerge. INT J CANCER. [Journal Article; Research Support, Non-U.S. Gov't; Review]. 2006 2006-1015;119(8):1755-60.

12. Batsakis JG. Nerves and neurotropic carcinomas. Ann Otol Rhinol Laryngol. [Journal Article]. 1985 1985-07-01;94(4 Pt 1):426-7.

13. Saidak Z, Clatot F, Chatelain D, Galmiche A. A gene expression profile associated with perineural invasion identifies a subset of HNSCC at risk of post-surgical recurrence. ORAL ONCOL. [Journal Article; Research Support, Non-U.S. Gov't]. 2018 2018-11-01;86:53-60.

14. Sun Q, Liu T, Liu P, Luo J, Zhang N, Lu K, et al. Perineural and lymphovascular invasion predicts for poor prognosis in locally advanced rectal cancer after neoadjuvant chemoradiotherapy and surgery. J CANCER. [Journal Article]. 2019 2019-01-20;10(10):2243-9.

15. Al-Sukhni E, Attwood K, Gabriel EM, LeVea CM, Kanehira K, Nurkin SJ. Lymphovascular and perineural invasion are associated with poor prognostic features and outcomes in colorectal cancer: A retrospective cohort study. INT J SURG. [Journal Article]. 2017 2017-01-01;37:42-9.

16. Cienfuegos JA, Martinez P, Baixauli J, Beorlegui C, Rosenstone S, Sola JJ, et al. Perineural Invasion is a Major Prognostic and Predictive Factor of Response to Adjuvant Chemotherapy in Stage I-II Colon Cancer. ANN SURG ONCOL. [Journal Article]. 2017 2017-04-01;24(4):1077-84.

17. Barresi V, Reggiani BL, Vitarelli E, Di Gregorio C, Ponz DLM, Barresi G. Immunohistochemical assessment of lymphovascular invasion in stage I colorectal carcinoma: prognostic relevance and correlation with nodal micrometastases. AM J SURG PATHOL. [Journal Article]. 2012 2012-0101;36(1):66-72.

18. Zhong JW, Yang SX, Chen RP, Zhou YH, Ye MS, Miao L, et al. Prognostic Value of Lymphovascular Invasion in Patients with Stage III Colorectal Cancer: A Retrospective Study. Med Sci Monit. [Journal Article]. 2019 2019-08-13;25:6043-50.

19. Fujita S, Nakanisi Y, Taniguchi H, Yamamoto S, Akasu T, Moriya Y, et al. Cancer invasion to Auerbach's plexus is an important prognostic factor in patients with pT3-pT4 colorectal cancer. DIS COLON RECTUM. [Journal Article]. 2007 2007-11-01;50(11):1860-6.

20. Merkel S, Mansmann U, Papadopoulos T, Wittekind C, Hohenberger W, Hermanek P. The prognostic inhomogeneity of colorectal carcinomas Stage III: a proposal for subdivision of Stage III. CANCERAM CANCER SOC. [Comparative Study; Journal Article]. 2001 2001-12-01;92(11):2754-9.

21. Mirkin KA, Hollenbeak CS, Mohamed A, Jia Y, El-Deiry WS, Messaris E. Impact of perineural invasion on survival in node negative colon cancer. CANCER BIOL THER. [Journal Article]. 2017 2017-0902;18(9):740-5.

22. Skancke M, Arnott SM, Amdur RL, Siegel RS, Obias VJ, Umapathi BA. Lymphovascular Invasion and Perineural Invasion Negatively Impact Overall Survival for Stage II Adenocarcinoma of the Colon. DIS 
COLON RECTUM. [Journal Article; Video-Audio Media]. 2019 2019-02-01;62(2):181-8.

23. Knijn N, Mogk SC, Teerenstra S, Simmer F, Nagtegaal ID. Perineural Invasion is a Strong Prognostic Factor in Colorectal Cancer: A Systematic Review. AM J SURG PATHOL. [Journal Article; MetaAnalysis; Research Support, Non-U.S. Gov't; Review; Systematic Review]. 2016 2016-01-01;40(1):10312.

24. Jiang HH, Zhang ZY, Wang XY, Tang X, Liu HL, Wang AL, et al. Prognostic significance of lymphovascular invasion in colorectal cancer and its association with genomic alterations. World $\mathrm{J}$ Gastroenterol. [Journal Article]. 2019 2019-05-28;25(20):2489-502.

25. Huh JW, Kim HR, Kim YJ. Lymphovascular or perineural invasion may predict lymph node metastasis in patients with T1 and T2 colorectal cancer. J GASTROINTEST SURG. [Journal Article; Research Support, Non-U.S. Gov't]. 2010 2010-07-01;14(7):1074-80.

26. Hogan J, Chang KH, Duff G, Samaha G, Kelly N, Burton M, et al. Lymphovascular invasion: a comprehensive appraisal in colon and rectal adenocarcinoma. DIS COLON RECTUM. [Comparative Study; Journal Article]. 2015 2015-06-01;58(6):547-55.

27. Lim SB, Yu CS, Jang SJ, Kim TW, Kim JH, Kim JC. Prognostic significance of lymphovascular invasion in sporadic colorectal cancer. DIS COLON RECTUM. [Journal Article; Research Support, NonU.S. Gov't]. 2010 2010-04-01;53(4):377-84.

28. Liebig C, Ayala G, Wilks J, Verstovsek G, Liu H, Agarwal N, et al. Perineural invasion is an independent predictor of outcome in colorectal cancer. J CLIN ONCOL. [Journal Article; Research Support, NonU.S. Gov't]. 2009 2009-11-01;27(31):5131-7.

29. Kim JC, Ha YJ, Tak KH, Roh SA, Kwon YH, Kim CW, et al. Opposite functions of GSN and OAS2 on colorectal cancer metastasis, mediating perineural and lymphovascular invasion, respectively. PLOS ONE. [Journal Article; Research Support, Non-U.S. Gov't]. 2018 2018-01-20;13(8):e202856.

30. Leijssen L, Dinaux AM, Taylor MS, Deshpande V, Kunitake H, Bordeianou LG, et al. Perineural Invasion Is a Prognostic but not a Predictive Factor in Nonmetastatic Colon Cancer. DIS COLON RECTUM. [Journal Article]. 2019 2019-10-01;62(10):1212-21.

31. Auclin E, Zaanan A, Vernerey D, Douard R, Gallois C, Laurent-Puig P, et al. Subgroups and prognostication in stage III colon cancer: future perspectives for adjuvant therapy. ANN ONCOL. [Journal Article; Review]. 2017 2017-05-01;28(5):958-68.

32. Murcia O, Juarez M, Rodriguez-Soler M, Hernandez-Illan E, Giner-Calabuig M, Alustiza M, et al. Colorectal cancer molecular classification using BRAF, KRAS, microsatellite instability and CIMP status: Prognostic implications and response to chemotherapy. PLOS ONE. [Journal Article; Observational Study; Research Support, Non-U.S. Gov't]. 2018 2018-01-20;13(9):e203051.

\section{Figures}



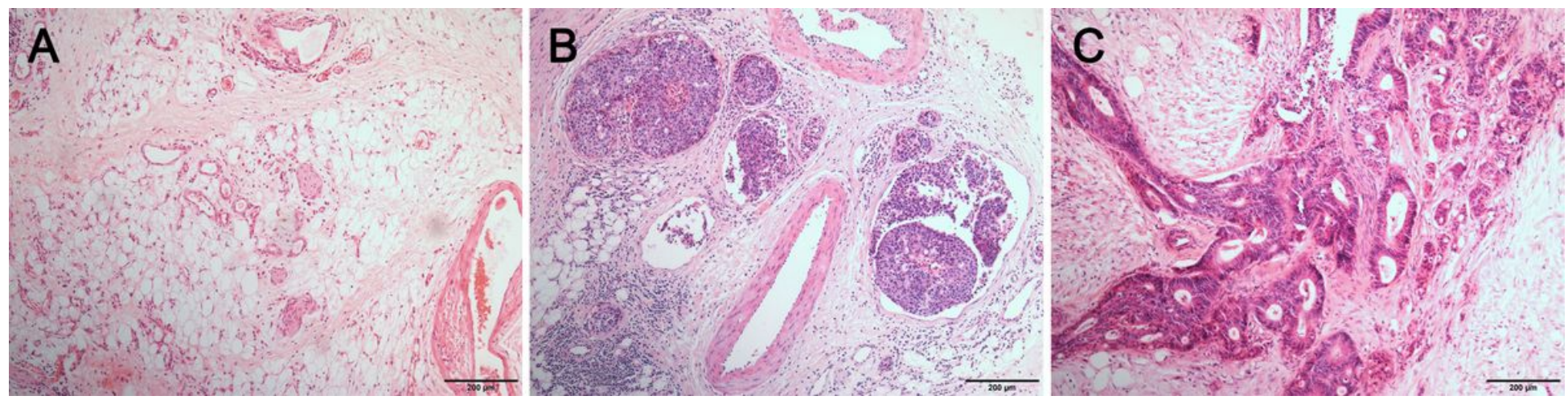

\section{Figure 1}

PNI was diagnosed as tumor invasion in, around, and through nerves and nerve sheaths (Figure 1)
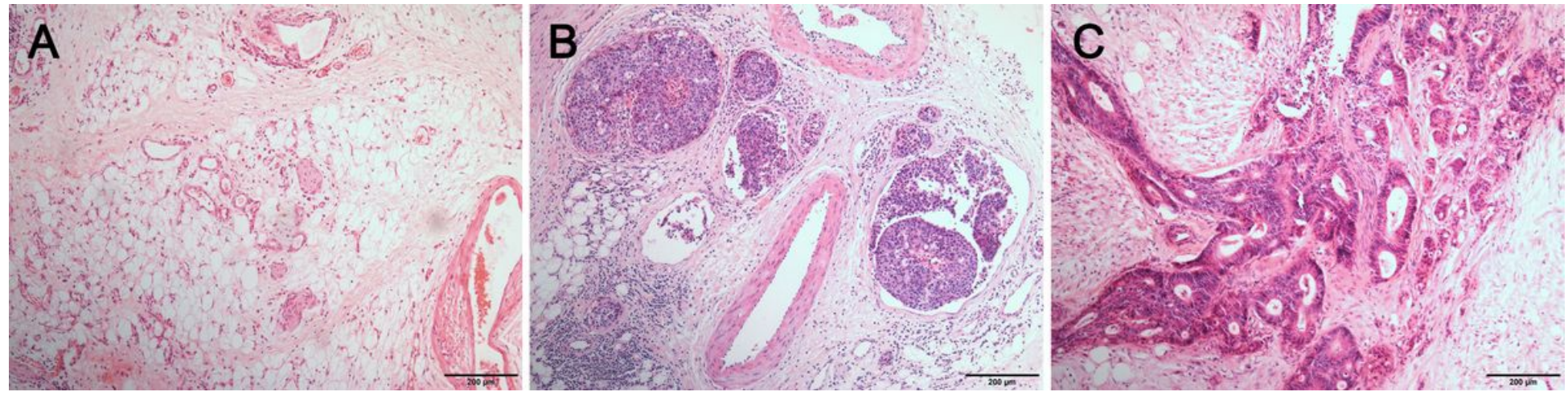

\section{Figure 1}

PNI was diagnosed as tumor invasion in, around, and through nerves and nerve sheaths (Figure 1) 

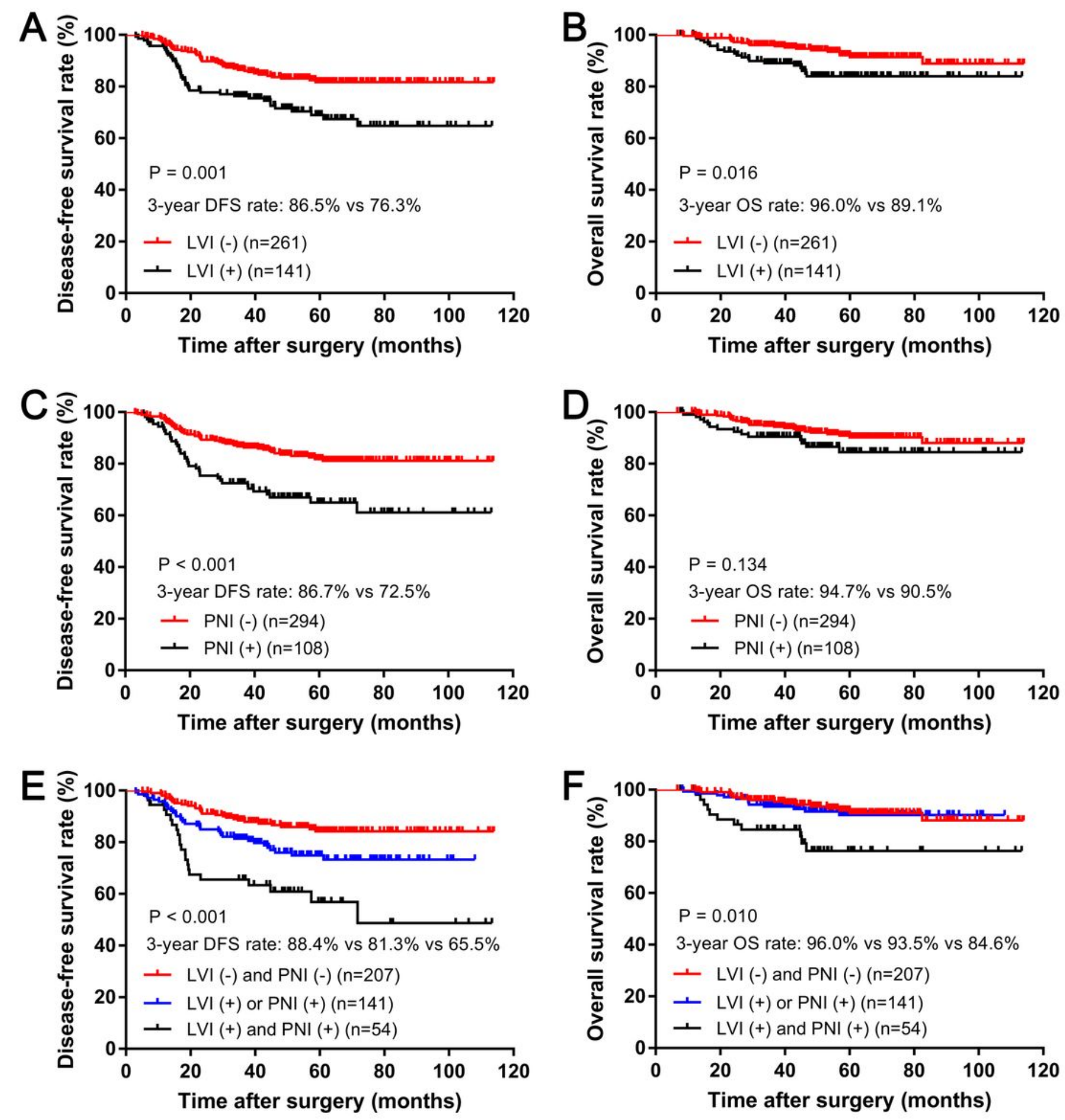

Figure 2

Kaplan-Meier analysis indicated that LVI-positive group showed the significantly worse 3-year DFS and OS rates compared with those in the LVI-negative group (DFS: $76.3 \%$ vs. $86.5 \%, P=0.001$, Figure $2 A$; OS: $89.1 \%$ vs. $96.0 \%, P=0.016$, Figure $2 B$ ). PNI-positive group presented significantly lower 3-year DFS rate by comparison of those with the PNI-negative group $(72.5 \%$ vs. $86.7 \%, \mathrm{P}<0.001$, Figure $2 \mathrm{C})$, while the 3-year OS rate was comparable between the two groups ( $90.5 \%$ vs. $94.7 \%, P=0.134$, Figure $2 \mathrm{D})$. Patients with the 

2E) and OS (84.6\% vs. 93.5\% vs. 96.0\%, P=0.010; Figure 2F) rates among those with either the presence of LVI and PNI and the absence of LVI and PNI.
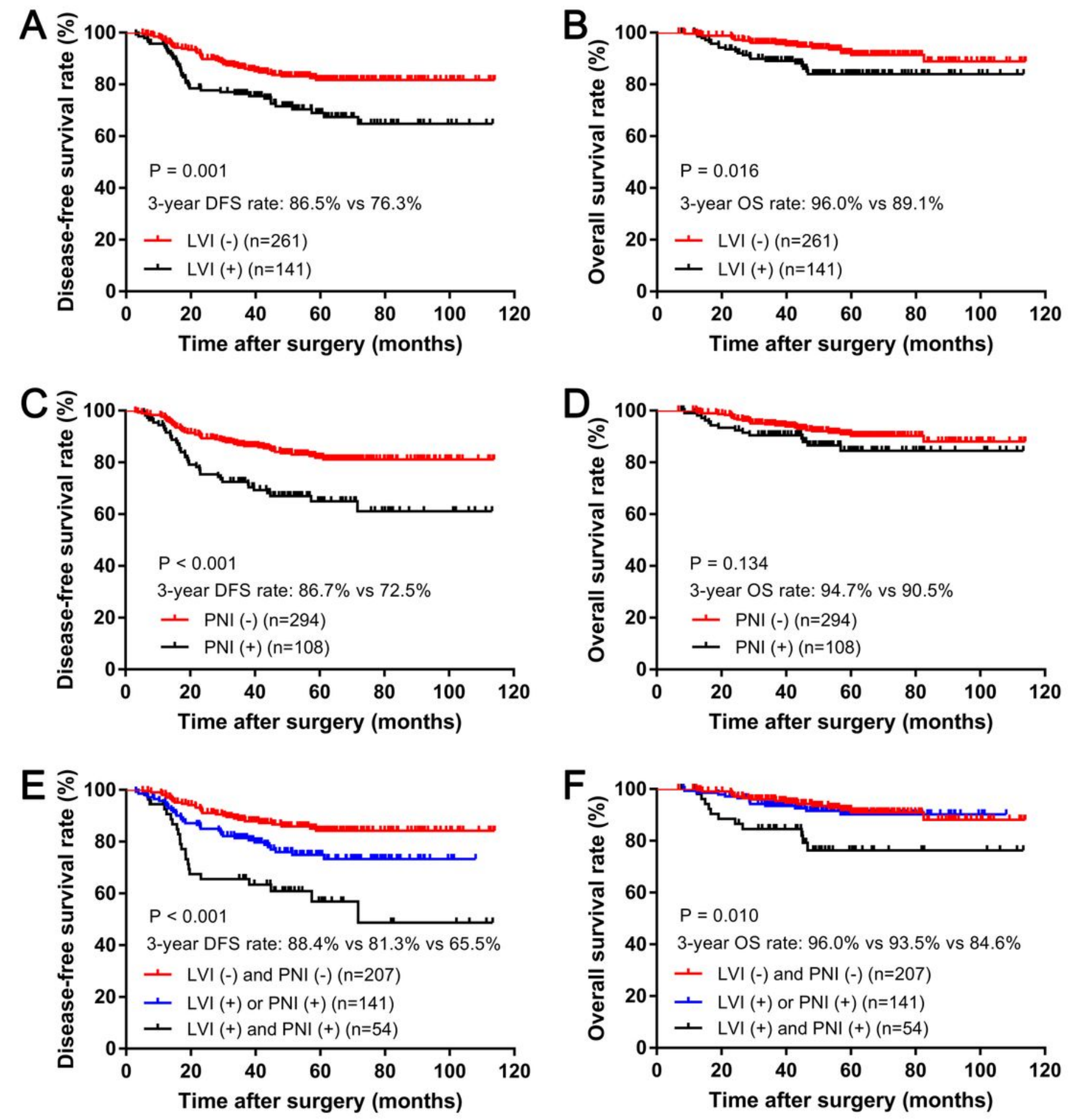

Figure 2

Kaplan-Meier analysis indicated that LVI-positive group showed the significantly worse 3-year DFS and OS rates compared with those in the LVI-negative group (DFS: $76.3 \%$ vs. $86.5 \%, P=0.001$, Figure 2A; OS: 
$89.1 \%$ vs. $96.0 \%, \mathrm{P}=0.016$, Figure $2 \mathrm{~B}$ ). PNI-positive group presented significantly lower 3-year DFS rate by comparison of those with the PNI-negative group (72.5\% vs. $86.7 \%, P<0.001$, Figure 2 C), while the 3-year OS rate was comparable between the two groups ( $90.5 \%$ vs. $94.7 \%, P=0.134$, Figure $2 D)$. Patients with the concurrent presence of LVI and PNI had the worst 3-year DFS (65.5\% vs. $81.3 \%$ vs. $88.4 \%, P<0.001$; Figure $2 \mathrm{E})$ and $\mathrm{OS}(84.6 \%$ vs. $93.5 \%$ vs. $96.0 \%, \mathrm{P}=0.010$; Figure $2 \mathrm{~F})$ rates among those with either the presence of LVI and PNI and the absence of LVI and PNI.
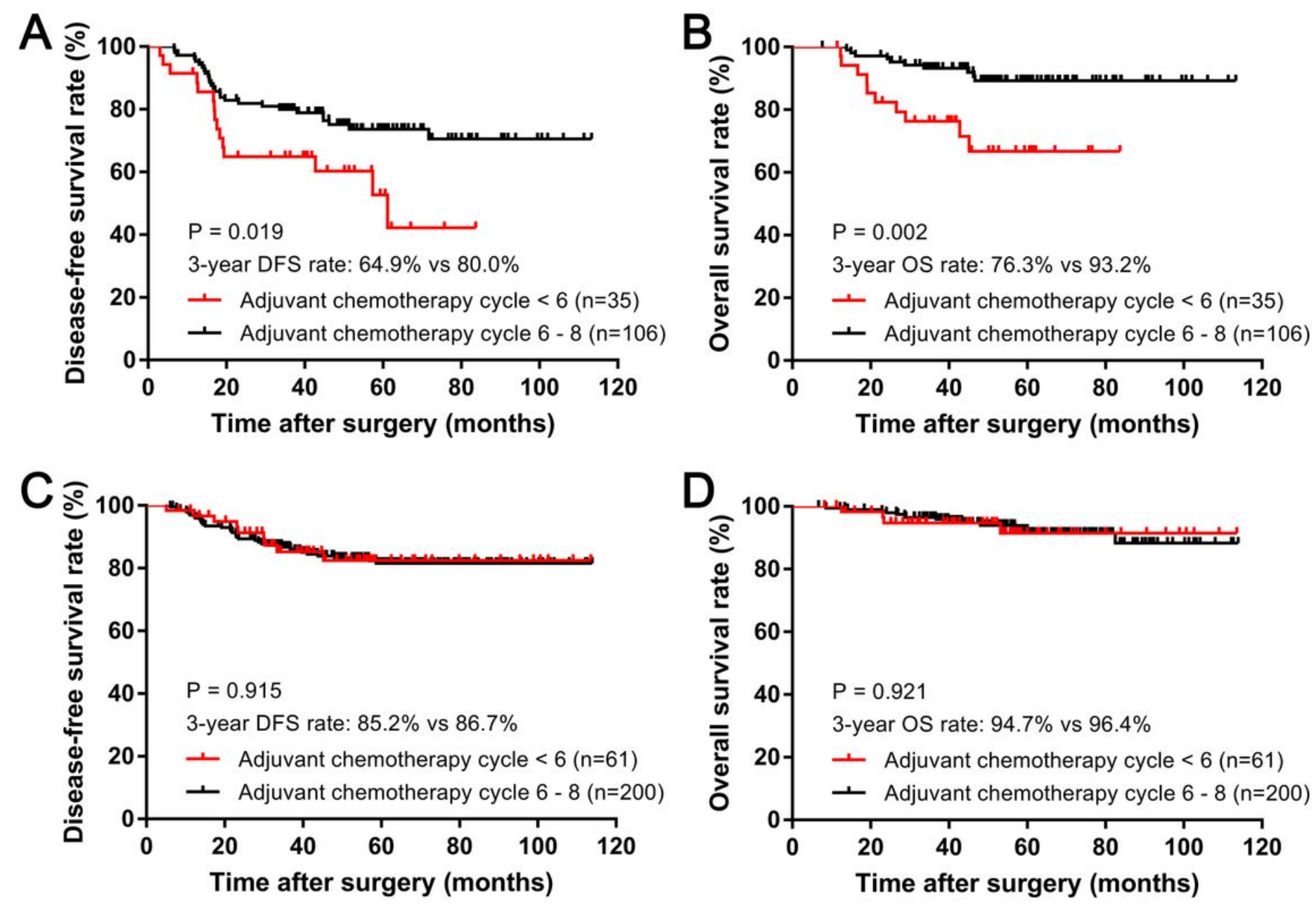

Figure 3

Among the LVI-positive group, the patients completing 6-8 cycles of adjuvant chemotherapy presented significantly better 3-year DFS and OS rates than those who completed less than 6 cycles (DFS: $80.0 \%$ vs. $64.9 \%, P=0.019$, Figure $3 A$; OS: $93.2 \%$ vs. $76.3 \%, P=0.002$, Figure $3 B$ ). 

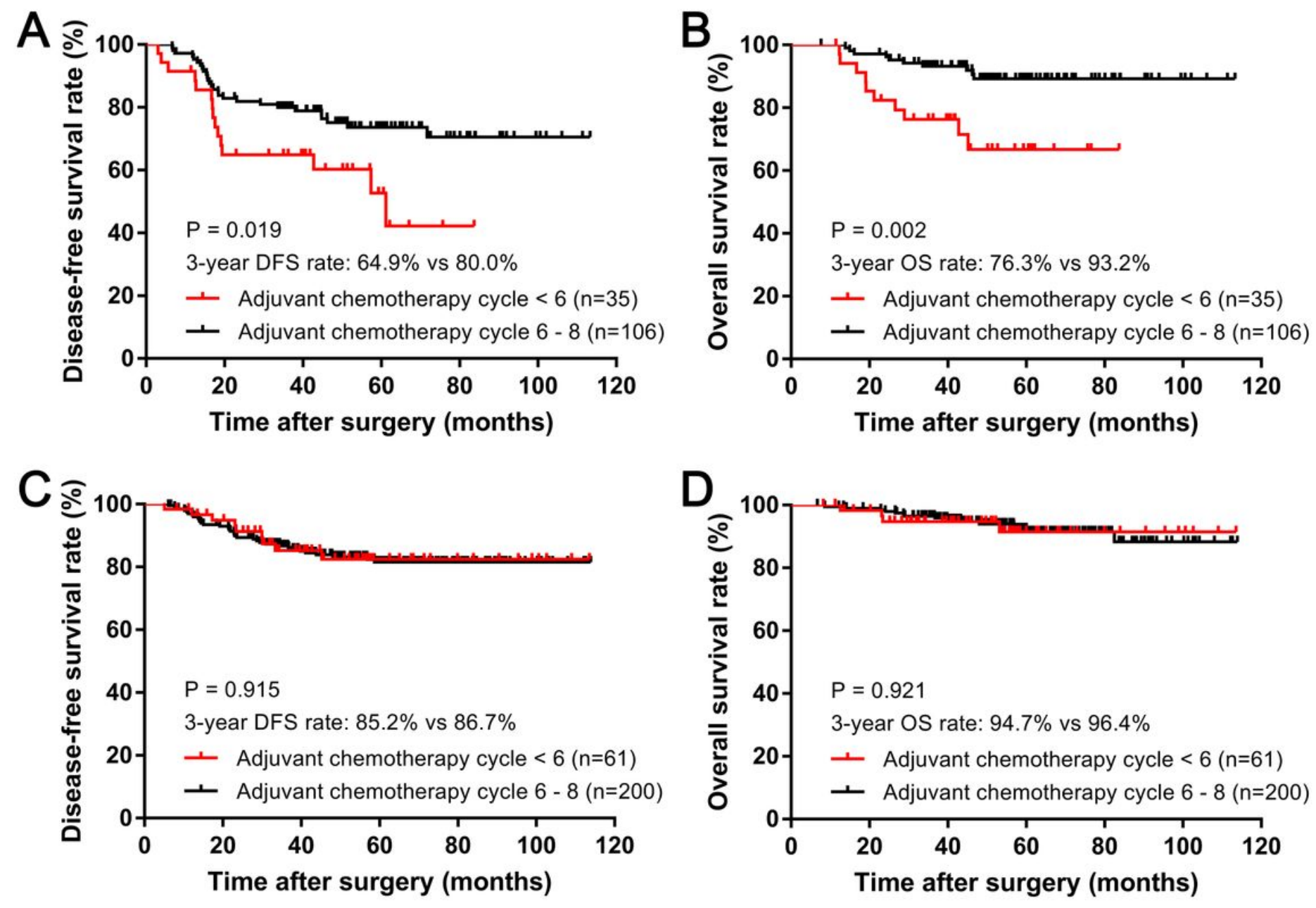

Figure 3

Among the LVI-positive group, the patients completing 6-8 cycles of adjuvant chemotherapy presented significantly better 3-year DFS and OS rates than those who completed less than 6 cycles (DFS: $80.0 \%$ vs. $64.9 \%, P=0.019$, Figure $3 A ; 0 S: 93.2 \%$ vs. $76.3 \%, P=0.002$, Figure $3 B$ ). 

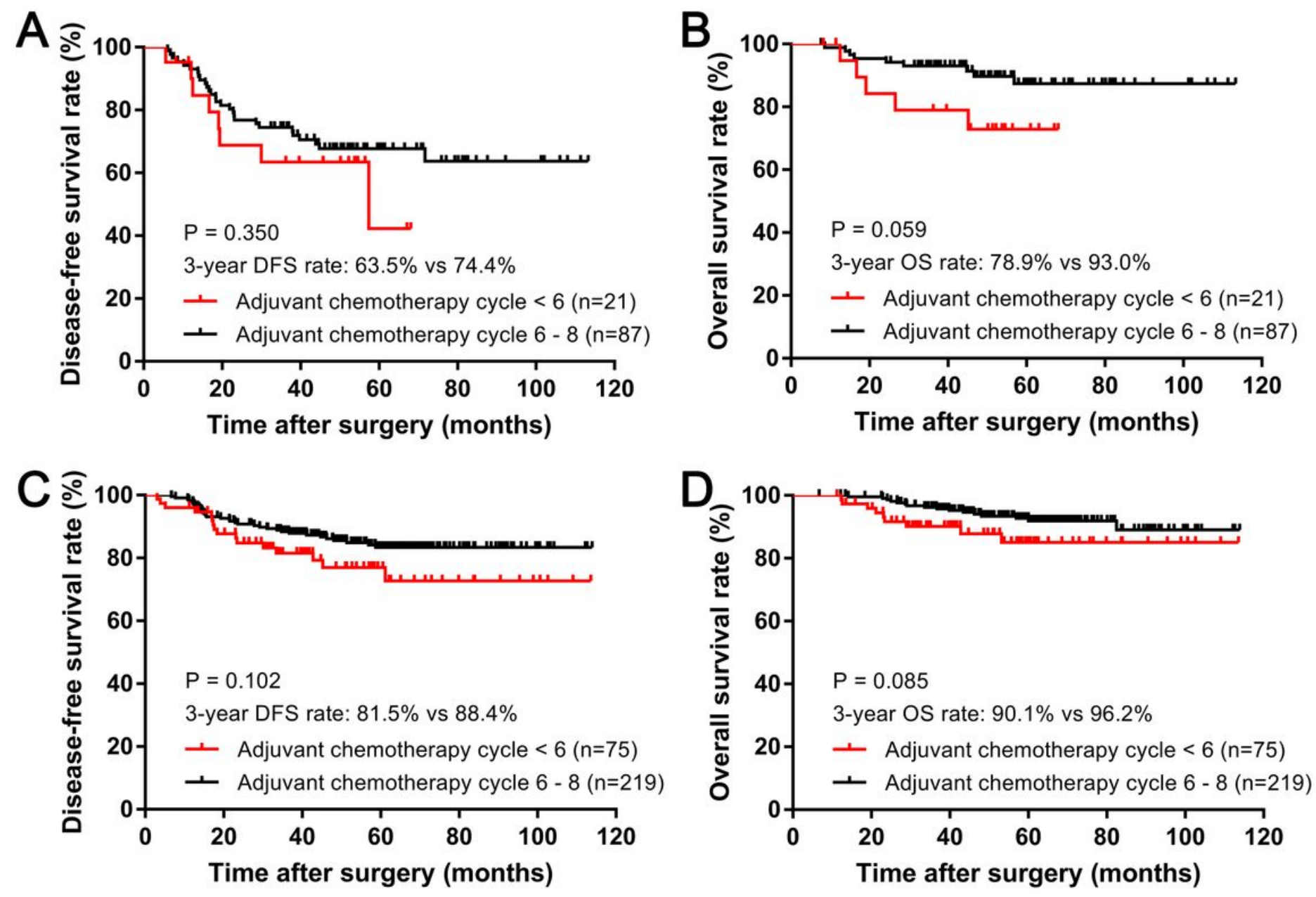

\section{Figure 4}

However, despite the PNI status, no significantly difference was observed in the 3-year DFS or OS rate whatever the patients completed 6-8 cycles of adjuvant chemotherapy or less than 6 cycles (Figure 4). 

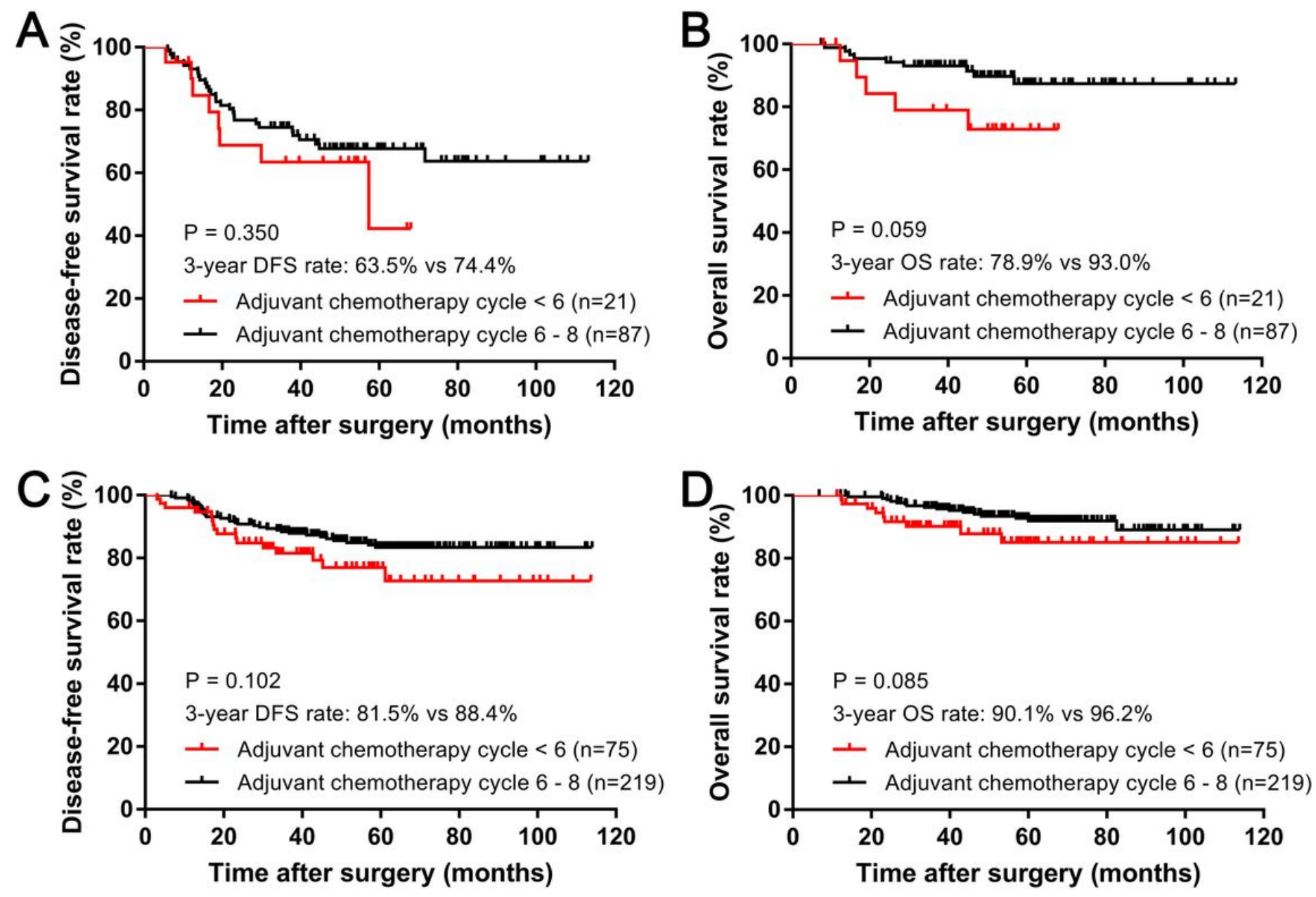

\section{Figure 4}

However, despite the PNI status, no significantly difference was observed in the 3-year DFS or OS rate whatever the patients completed 6-8 cycles of adjuvant chemotherapy or less than 6 cycles (Figure 4). 